\title{
Impact des recommandations de l'ANSM dans la prise en charge en cabinet libéral des collections circonscrites aiguës suppurées de la cavité orale d'origine dentaire : enquête nationale
}

\author{
E. Noaillon (Claye Souilly), S. Azogui-Lévy (Paris), G. Lescaille (Paris), R. Toledo (Paris), V. \\ Descroix (Paris), P. Goudot (Paris), J. Rochefort (Paris)
}

Introduction: La prise en charge des collections circonscrites aiguës suppurées d'origine dentaire (CCASD) implique un drainage, le traitement de la dent causale ainsi que la mise en place d'une antibiothérapie dont le choix repose actuellement sur les recommandations de l'ANSM (recommandations AFSSAPS, 2011). En première intention, elles préconisent la prescription d'amoxicilline $(2 \mathrm{~g} / \mathrm{jours})$ ou clindamycine (1200g/jour) en cas d'allergie; en seconde intention, une bithérapie associant classiquement l'amoxicilline à l'acide clavulanique ou au métronidazole. L'émergence de mécanismes de résistances bactériennes a conduit à la mise en place d'actions favorisant une meilleure utilisation des antibiotiques. La France s'inscrit parmi les plus gros consommateurs en Europe (ANSM 2017): une surconsommation globale, une utilisation trop fréquente de molécules à large spectre en pratique courante. Dans ce contexte, l'ANSM a émis des recommandations sur la prescription des antibiotiques pour la prise en charge des CCASD. Sachant que $90 \%$ des prescriptions sont faites en ville dont $8 \%$ par les chirurgiens-dentistes (CD), il est paru nécessaire de connaître leur attitude. Nous avons donc souhaité évaluer l'impact des recommandations de l'ANSM chez les CD en France, dans la prise en charge des CCASD. Matériel et méthode: Nous avons réalisé une enquête nationale, en collaboration avec l'UFSBD (Union Française pour la Santé Bucco-Dentaire), regroupant 15000 praticiens, ayant reçu le questionnaire par mail. Il comprenait 28 questions sur quatre thèmes principaux: prise en charge des CCASD, connaissance des recommandations, leur application, formations professionnelles initiales et continues. Résultats: Sur l'ensemble des praticiens, $690(4,6 \%)$ ont participé à l'étude. 13\% suivent de manière stricte les recommandations et 70,5\% réalisent un acte clinique le jour de l'urgence : il correspond à $98 \%$ à un drainage (par voie muqueuse ou ouverture de la dent). Dans seulement $1 / 3$ des cas la prescription de première intention se portait sur l'amoxicilline seule. $44 \%$ y associent des anti-inflammatoires. $68 \%$ des praticiens reconnaissent connaitre les recommandations de l'ANSM par leur formation initiale $(36 \%)$, une revue professionnelle $(27 \%)$ ou un congrès $(19 \%)$. La plupart suit une formation continue $(77 \%$ une revue professionnelle, $90 \%$ un congrès, $38 \%$ un cursus de formation continue théorique). 89\% prescrivent des anti-inflammatoires lors de la consultation d'urgence. Conclusion: Il existe peu de données évaluant l'impact des recommandations concernant ce sujet, mais il est communément admis qu'elles sont peu suivies (Gaillat et al 2005), ce que révèle également notre étude. Les raisons invoquées sont multiples : désaccord des praticiens sur l'efficacité des recommandations, manque de temps, contraintes organisationnelles. Bien que la bithérapie soit considérée comme plus efficace par les CD interrogés pour traiter les CCASD, les experts s'accordent à dire qu'elle participe à la création de la résistance bactérienne, considérée comme «l'une des plus graves menaces pesant sur la santé mondiale », et faisant l'objet d'un plan d'action mondial mis en place par l'OMS et l'ONU en 2015 (OMS, 2017). 